\title{
Trypophobic Discomfort is Spatial-Frequency Dependent
}

\author{
Kyoshiro Sasaki ${ }^{1,2,3}$, Yuki Yamada ${ }^{2}$, Daiichiro Kuroki ${ }^{4}$, and Kayo Miura ${ }^{5}$ \\ ${ }^{1}$ Faculty of Science and Engineering, Waseda University, Tokyo, Japan \\ ${ }^{2}$ Faculty of Arts and Science, Kyushu University, Fukuoka, Japan \\ ${ }^{3}$ Japan Society for the Promotion of Science, Kojimachi Business Center Building, Tokyo, Japan \\ ${ }^{4}$ School of Letters, Kyushu University, Fukuoka, Japan \\ ${ }^{5}$ Faculty of Human-Environment Studies, Kyushu University, Fukuoka, Japan
}

ABSTRACT

Clusters of holes, such as those in a lotus seedpod, induce trypophobic discomfort. Previous research has demonstrated that high-contrast energy at midrange spatial frequencies in images causes trypophobic discomfort. The present study examined the effects on discomfort of eliminating various spatial frequency components from the images to reveal how each spatial frequency contributes to the discomfort. Experiment 1 showed that eliminating midrange spatial frequencies did not affect trypophobic discomfort, while Experiment 2 revealed that images of holes that consisted of only high-spatial frequencies evoked less discomfort than other images and that images containing only low or midrange spatial frequencies induced as much trypophobic discomfort as did the original images. Finally, Experiment 3 found that participants with a high level of the trypophobic trait experienced stronger discomfort from the original images and the images containing only low or midrange spatial frequencies than participants with a low level of the trypophobic trait. Our findings thus suggest that trypophobic discomfort can be induced by middle and low spatial

frequencies.

trypophobia,

spatial frequency,

emotion

\section{INTRODUCTION}

Humans may experience an unpleasant sensation when confronted with certain visual scenes. For instance, some situations (such as a dirty toilet), animals (such as spiders and birds), or wounded people can induce strong negative emotions (Caseras et al., 2007; Curtis, Aunger, \& Rabie, 2004; Sawchuk, Lohr, Westendorf, Meunier, \& Tolin, 2002). Humans also respond negatively to objects that are difficult to categorize (Kawabe, Sasaki, Ihaya, \& Yamada, 2017; Yamada, Kawabe, \& Ihaya, 2012, 2013; Yamada, Sasaki, Kunieda, \& Wada, 2014). These observations suggest the existence of a system to avoid things that may be perceived as unpleasant. Such a system would be a beneficial way to estimate whether objects are potentially harmful without touching them directly. If the objects are dangerous, we might not survive when interacting directly with them; thus, the proposed system uses sensory information to prevent us from touching or eating dangerous objects.
A recent study has reported that clusters of holes can induce pathological discomfort or fear (trypophobia; Cole \& Wilkins, 2013). Cole and Wilkins (2013) also suggested that clusters of round objects, as well as holes, induce the same discomfort and normal observers may experience trypophobic discomfort. They investigated why clusters of holes or round objects make normal observers experience unpleasant feelings by performing a spectral analysis of trypophobic images

Corresponding authors: Kyoshiro Sasaki, Faculty of Science and Engineering, Waseda University, 3-4-1, Ohkubo, Shinjuku, Tokyo, 169-8555, Japan. Phone \& Fax:+81-3-5286-3335. Email: kyoshiro0920@gmail.com

Yuki Yamada, Faculty of Arts and Science, Kyushu University, 744 Motooka, Nishi-ku, Fukuoka, 819-0395, Japan. Phone \& Fax: +81-92-802-5837 Email: yamadayuk@gmail.com 
(such as a lotus seedpod) and control images (such as a golf cup) and found that the contrast energy with midrange spatial frequencies was higher in the trypophobic images than in the control images. This spectral characteristic was consistent with that of potentially dangerous or poisonous animals (such as snakes), images of which also have high-contrast energy with midrange spatial frequencies in comparison with those of nonhazardous animals. Additionally, a study of visual perception suggested that high-contrast energy at midrange spatial frequencies induces visual discomfort (Fernandez \& Wilkins, 2008). Thus, these findings indicate that contrast energy at midrange spatial frequencies is important for inducing trypophobia.

A previous study by Le, Cole, and Wilkins (2015) provided evidence challenging the indication that trypophobic discomfort can be explained only by the contrast energy at midrange spatial frequencies. They examined whether their previous observations from spectral analyses (Cole \& Wilkins, 2013) were plausible. Images that deviated from the statistical norm of natural images, which have a 1/famplitude spectrum, caused discomfort (e.g., Fernandez \& Wilkins, 2008; O’Hare \& Hibbard, 2011). Additionally, as previously stated, the spectral analysis revealed that trypophobic images had excess contrast energy at midrange frequencies compared to natural images (Cole \& Wilkins, 2013). Based on these studies, Le et al. examined whether discomfort from trypophobic images was reduced by filtering those images such that they had $1 / f$ amplitude spectra and removing excess energy. Their results showed that the trypophobic images still induced discomfort, even when the excess energy at midrange spatial frequencies was removed. These findings indicate that trypophobic discomfort is induced not only by excess contrast energy at midrange frequencies, but also by several other factors.

The present study aimed to reveal more precisely how the spatial frequency of the stimulus is related to trypophobic discomfort. Spectral analyses have indicated that midrange spatial frequencies are crucial in inducing trypophobic discomfort (Cole \& Wilkins, 2013). However, the results of manipulations of contrast energy in the middle range are not consistent with this idea (Le et al., 2015). Le et al. (2015) suggested that the spatial frequency components at other ranges also contribute to trypophobic discomfort. The present study examined the effects of eliminating spatial frequency components at various ranges on trypophobic discomfort. By exploring whether discomfort was modulated by eliminating the spatial frequency components at each range, we hoped to identify which ranges of spatial frequency components are involved in trypophobic discomfort.

\section{EXPERIMENT 1}

\section{Methods}

\section{PARTICIPANTS}

Fifteen volunteers participated in the experiment (10 males and five females, $M_{\text {age }} \pm S E M=22.5 \pm 0.65$ ). All participants were naive to the aim of this experiment and reported that they had normal vi- sion. The study protocol was approved by the ethics committees of Kyushu University. The experiment was conducted according to the guidelines stipulated in the Declaration of Helsinki. We obtained written informed consent from all participants prior to commencement of data collection.

\section{APPARATUS}

The stimuli were presented on a 22 in., gamma-corrected cathode ray tube monitor (Mitsubishi RDF225WG, Mitsubishi Electric Corporation). The resolution was $1,024 \times 768$ pixels, and the refresh rate was $100 \mathrm{~Hz}$. The presentation of the stimuli and the collection of data were controlled by a computer (Apple Mac Pro, Apple). The stimuli were generated by MATLAB (The MathWorks, Inc.) with the Psychtoolbox extension (Brainard, 1997; Pelli, 1997).

\section{STIMULI}

The viewing distance was $57 \mathrm{~cm}$. The stimuli comprised a fixation mark, cursors for rating, and visual images. The fixation mark consisted of two white concentric circles $\left(0.29^{\circ}\right.$ and $0.17^{\circ}$ radii; $91 \mathrm{~cd} / \mathrm{m}^{2}$ luminance). The cursors were white boxes that surrounded each rating value, and the selected box was deep gray.

The stimuli were 20 trypophobic and 20 control images $(512 \times 512$ pixels; $20^{\circ} \times 20^{\circ}$ ) that were used in Experiment 2 of Le et al. (2015). The trypophobic images consisted of clusters of holes and round objects such as lotus seedpods, barnacles, and so on. Le et al. took the trypophobic images from www.trypophobia.com and a Facebook group page (Trypophobia: Fear of Clusters of Holes, https://www.facebook.com/ groups/3318322299/; accessed on February 24, 2017). None of the trypophobic images were artificially manipulated. Le et al. also conducted a Google image search for objects with holes (such as a guitar case and trumpet), which they then used as control images. These images were not present on the trypophobia websites and were neutral. We used Photoshop (Adobe Systems Incorporated) to normalize the images' gray levels (mean gray level $=125$ ) and applied a Hanning Window to them. As was done by Cole and Wilkins (2013), we conducted spectral analyses, and the results showed that the contrast energy, ranging from 45 cycles per image (cpi) to $181 \mathrm{cpi}$ (that is, from 2.25 cycles per degree [cpd] to $9.05 \mathrm{cpd}$ ), was significantly higher in the trypophobic

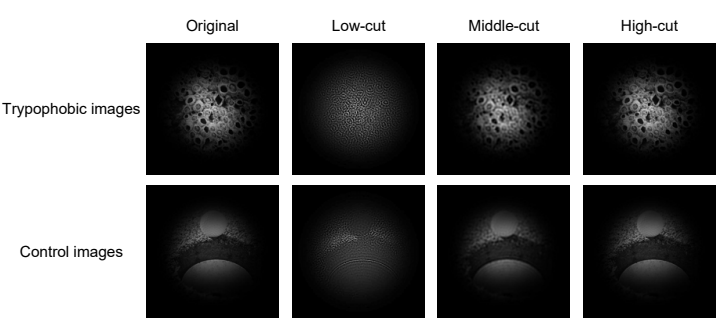

\section{FIGURE 1.}

Examples of the images used in Experiment 1.These images were optimized for presentation on a gamma-corrected monitor. For visibility on nongamma-corrected monitors, the luminance of the images has been slightly adjusted in this figure. 
images than in the control images $(p<.003 \text {, for all cases })^{1}$. There were also significant differences in the contrast energies at 5 and 8 cpi $(0.25$ and $0.4 \mathrm{cpd}, p<.003$, for both cases), while the contrast energies were higher in the control images than in the trypophobic images at these points, unlike at the points from 45 to $181 \mathrm{cpi}$. We first eliminated the spatial frequency components within the range of 45-181 cpi (middlecut), and the spatial frequency components under $45 \mathrm{cpi}$ (low-cut) and over $181 \mathrm{cpi}$ (high-cut). Figure 1 shows examples of the stimuli used in Experiment 1 . In all of the images, we retained the constant components $(0 \mathrm{cpi})$ that were involved in their average luminance.

\section{PROCEDURE}

The experiment was conducted in a darkened room. The participants started each trial by pressing the spacebar on a computer keyboard. After the fixation mark was presented for $500 \mathrm{~ms}$, the image stimulus and rating cursors were presented, and they remained until the evaluation was made. The participants were asked to evaluate their degree of discomfort for each image on an 11-point scale that ranged from -5 (strong discomfort) to 5 (strong comfort). Each participant performed 160 trials: two image types (trypophobic and control) $\times$ four elimination ranges (original, low-cut, middle-cut, and high-cut) $\times 20$ stimuli. Trials were randomized for each participant.

\section{Results and Discussion}

Figure 2 shows a bar graph illustrating the evaluations of discomfort for each of the image types and four eliminated frequency ranges. We conducted a two-way within-participant analysis of variance (ANOVA) with Image Type and Eliminated Ranges as factors. The results revealed significant main effects of image type, $F(1,14)=73.72, p<.001, \eta_{\mathrm{p}}{ }^{2}=$ .84 , and elimination range, $F(3,42)=12.04, p<.001, \eta_{\mathrm{p}}{ }^{2}=.46$. The interaction between these factors was also significant, $F(3,42)=21.88$,

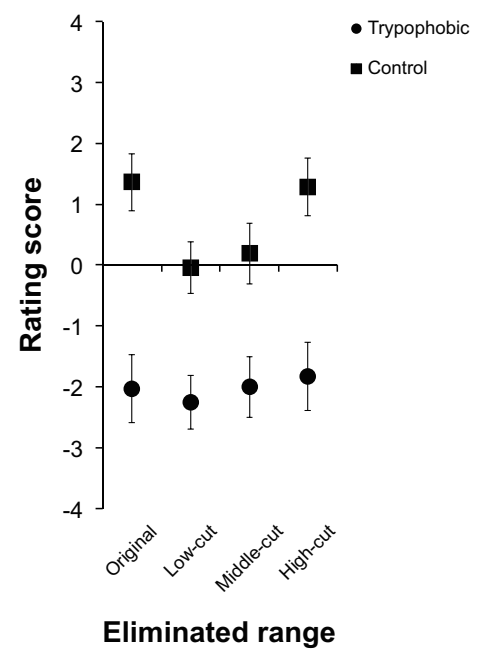

FIGURE 2.

Results of Experiment 1. A bar graph illustrating the results of the evaluations of discomfort for each of the image types and four elimination ranges. The error bars denote $95 \%$ Cls. $p<.001, \eta_{\mathrm{p}}^{2}=.61$. Post-hoc tests revealed simple main effects of image type in the original, low-cut, middle-cut, and high-cut conditions, $F(1$, 14) $>48.16, p<.001, \eta_{\mathrm{p}}{ }^{2}>.77$, for all conditions. The simple main effect of elimination range was also significant in the control condition, $F(3$, $42)=20.87, p<.001, \eta_{p}{ }^{2}=.60$, while this effect was not significant in the trypophobic condition, $F(3,42)=2.16, p=.11, \eta_{p}{ }^{2}=.13$. For the control condition, multiple comparisons with Shaffer's (1986) method demonstrated that the rating scores were higher in the original and high-cut conditions than in the low-cut and middle-cut conditions, $t(14)>3.76, p<.007$, Cohen's $d>1.14$, for both conditions.

Furthermore, to confirm whether each image induced comfort or discomfort, we conducted one-sample $t$ tests between 0 and the rating scores of each condition. In the trypophobic condition, the rating scores of all conditions differed significantly from $0, t(14)>6.37, p<$ .001 , Cohen's $d>2.33$. In contrast, in the control condition, the rating scores of the original and high-cut conditions differed significantly from $0, t(14)>5.29, p<.001$, Cohen's $d>1.94$, while those of the lowcut and middle-cut conditions did not, $t(14)<0.75, p>.46$, Cohen's $d$ $<0.27$, for both conditions.

The results indicated that, as in previous studies (Cole \& Wilkins, 2013; Le et al., 2015), the trypophobic images induced discomfort. Furthermore, the results showed that eliminating the mid-frequency components did not reduce the level of discomfort associated with the trypophobic images. A previous study (Cole \& Wilkins, 2013) revealed that the contrast energy at midrange spatial frequencies was higher in the trypophobic images than in the control images, which indicated that this difference in contrast energy caused trypophobic discomfort. In contrast, Le et al. (2015) found that trypophobic discomfort was not reduced even when the excess energy was removed, and our findings were consistent with these results. The results of Experiment 1 and the previous study (Le et al., 2015) indicated that trypophobic discomfort was not based solely on the mid-frequency components. Experiment 1 investigated whether the elimination of the spatial frequency components at the low, middle, and high ranges affected trypophobic discomfort. In order to directly determine the ranges of the spatial frequency components that induced trypophobic discomfort, Experiment 2 examined the specific frequency components of the low, middle, and high ranges and measured trypophobic discomfort.

\section{EXPERIMENT 2}

\section{Methods}

\section{PARTICIPANTS, APPARATUS, STIMULI, AND PROCEDURE}

Fifteen volunteers participated in the experiment (nine males and six females, $M_{\text {age }} \pm S E M=23.9 \pm 0.72$ ). The methods were identical to those of Experiment 1, except that we used images that included only the low-, mid-, or high-frequency components (low-pass, middle-pass, or high-pass conditions, respectively) instead of images in which the spatial frequency components at each range were eliminated. Figure 3 shows examples of the stimuli that were used in Experiment 2. 


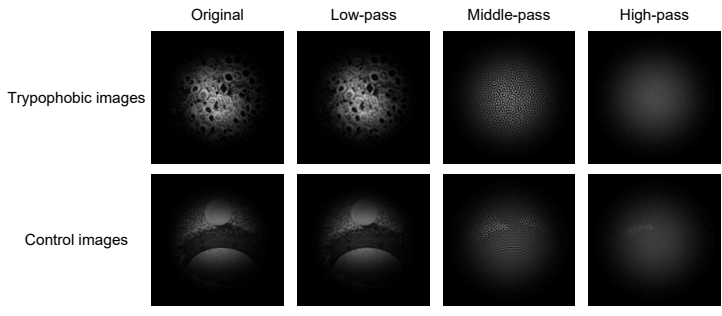

FIGURE 3.

Examples of the images used in Experiment 2. These images were optimized for presentation on a gamma-corrected monitor. For visibility on nongamma-corrected monitors, the luminance of the images has been slightly adjusted in this figure.

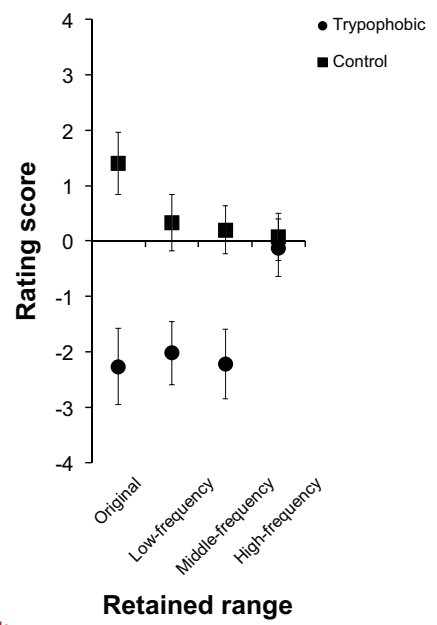

\section{FIGURE 4. Retained range}

Results of Experiment 2. A bar graph illustrating the results of the evaluations of discomfort for each of the image types and four retained ranges. The error bars denote $95 \%$ Cls.

\section{Results and Discussion}

Figure 4 shows a bar graph illustrating the evaluations of discomfort for each of the image types and four retained ranges. We conducted a two-way within-participant ANOVA with Image Type and Retained Range as factors. The results revealed a significant main effect of image type, $F(1,14)=67.91, p<.001, \eta_{\mathrm{p}}{ }^{2}=.83$, and retained range, $F(3,42)=$ $7.81, p<.001, \eta_{\mathrm{p}}{ }^{2}=.36$. The interaction between these factors was also significant, $F(3,42)=51.89, p<.001, \eta_{\mathrm{p}}{ }^{2}=.79$. Post-hoc tests revealed that the simple main effects of image type in the original, low-pass, middle-pass, and high-pass conditions were significant, $F(1,14)>4.64$, $p<.05, \eta_{\mathrm{p}}{ }^{2}>.24$, for all conditions. Moreover, the simple main effects of the retained range were significant in the trypophobic and control conditions, $F(3,42)>12.98, p<.001, \eta_{\mathrm{p}}{ }^{2}>.48$, for both conditions. Multiple comparisons with Shaffer's (1986) method demonstrated that in the trypophobic condition, the rating scores of the high-pass condition were significantly higher than those of the original, low-pass, and middle-pass conditions, $t(14)>5.21, p<.001$, Cohen's $d>1.76$, for all comparisons. In the control condition, the rating scores of the original condition differed significantly from the low-pass, middle-pass, and high-pass conditions, $t(14)>4.46, p<.002$, Cohen's $d>1$, for all comparisons.

Furthermore, we conducted one-sample $t$ tests between 0 and the rating scores of each condition. In the trypophobic condition, the rating scores of the original, low-pass, and middle-pass conditions differed significantly from $0, t(14)>6.40, p<.001$, Cohen's $d>2.34$, for all conditions, while the rating scores of the high-pass condition did not differ significantly from $0, t(14)=0.48, p=.64$, Cohen's $d=0.18$. In contrast, in the control condition, the rating scores of the original condition differed significantly from $0, t(14)=4.85, p<.001$, Cohen's $d$ $=1.77$, while those of the low-pass, middle-pass, and high-pass conditions did not significantly differ from $0, t(14)<1.29, p>.22$, Cohen's $d$ $<0.47$, for all conditions.

Experiment 2 revealed that the images in the original, low-pass, and middle-pass conditions induced comparable discomfort in the trypophobic condition. In contrast, the images in the high-pass condition did not induce discomfort. These findings suggested that the high-frequency components were not involved in trypophobic discomfort, whereas the low- and mid-frequency components were key factors in inducing this discomfort. However, it was unclear whether both the low- and mid-frequency components were the specific factors in trypophobic discomfort. A previous study has shown that midfrequency components are related to the visual discomfort induced by other types of image (Fernandez \& Wilkins, 2008). Thus, it is possible that mid-frequency components contribute only to general visual discomfort and that the specific factor that causes trypophobic discomfort exists only in the low-frequency components. To address these issues, Experiment 3 examined the relationship between individual differences in trypophobic trait and trypophobic discomfort using the original, low-pass, middle-pass, and high-pass images. Le et al. (2015) developed the Trypophobia Questionnaire (TQ), which can measure an observer's trypophobic trait. Le et al. have revealed that people with high TQ scores felt stronger discomfort from trypophobic images than people with low TQ scores. That is, people with high TQ scores (a high level of the trypophobic trait) tend to react to trypophobic images with stronger discomfort than people with low TQ scores (a low level of the trypophobic trait). Considering this TQ trait, if low-frequency components were the only key factors in trypophobic discomfort, people with a high level of the trypophobic trait (i.e., with relatively high TQ scores) should experience stronger discomfort from the original and low-pass trypophobic images than people with a low level of the trypophobic trait (i.e., with relatively low TQ scores), and the degree of discomfort from the middle-pass trypophobic images would not be different between levels of the trypophobic trait. However, if both low- and midfrequency components were responsible for trypophobic discomfort, people with a high level of the trypophobic trait should experience stronger discomfort from the original, low-pass, and middle-pass trypophobic images than people with a low level of the trypophobic trait. Moreover, based on the results of Experiment 2, which indicate that high-frequency components did not induce trypophobic discomfort, there would be no difference in discomfort from the high-pass trypophobic image between levels of the trypophobic trait. 


\section{EXPERIMENT 3}

\section{Methods}

\section{PARTICIPANTS, APPARATUS, STIMULI, AND PROCEDURE}

Before the laboratory experiment, we recruited 105 volunteers to respond to the Japanese version of the TQ (Chaya, Xue, Uto, Yao, \& Yamada, 2016). As in the original version of the TQ (Le et al., 2015), participants observed two trypophobic images (a lotus seed head and a honeycomb) and then responded to 17 items (e.g., "Vomit," "Have trouble breathing," and "Shiver"; for more detailed information, see Le et al., 2015) and two dummy items ("Feel at peace" and "Want to laugh"), according to the extent of their reaction to the items referred to when viewing the trypophobic images, on a 5 -point Likert scale $(1=$ not at all; $5=$ extremely). We then added the scores of all items, except for the dummy items, and asked volunteers with scores among the top and bottom $33 \%$ of the total scores to participate in the experiment, where higher TQ scores represent a higher trypophobic trait. As a result, 12 volunteers in the high TQ group and 10 in the low TQ group participated in Experiment 3 (six males and 16 females, $M_{\text {age }} \pm S E M=$ $22.4 \pm 0.52$ ). The TQ scores were significantly higher in the high $\mathrm{TQ}$ group than in the low TQ group, $t(20)=7.71, p<.001$, Cohen's $d=$ 3.30. The methods were identical to those used in Experiment 2.

\section{Results and Discussion}

Figure 5 shows a bar graph illustrating the evaluations of discomfort for each of the image types and the four retained ranges. We conducted a three-way mixed ANOVA with Image Type and Retained Range as within-participant factors and Trypophobic-trait Group as a betweenparticipants factor. The results revealed a significant main effect of try-

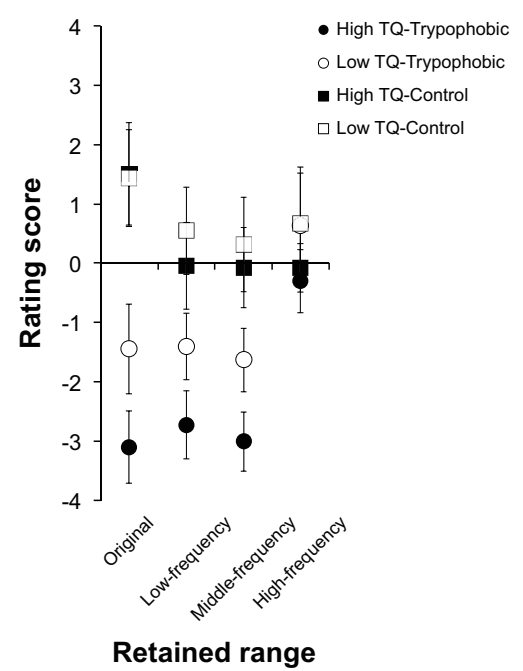

FIGURE 5.

Results of Experiment 3. A bar graph illustrating the results of the evaluations of discomfort for each of the trypophobic-trait groups, the image types, and four retained ranges. The error bars denote $95 \%$ Cls. TQ: Trypophobia Questionnaire. pophobic-trait group, $F(1,20)=7.07, p=.02, \eta_{\mathrm{p}}{ }^{2}=.26$, image type, $F(1$, $20)=85.91, p<.001, \eta_{\mathrm{p}}{ }^{2}=.81$, and retained range, $F(3,60)=16.61, p<$ $.001, \eta_{\mathrm{p}}{ }^{2}=.45$. The interaction between image type and retained range was also significant, $F(3,60)=66.36, p<.001, \eta_{\mathrm{p}}{ }^{2}=.77$. Importantly, the interaction among the three factors was significant, $F(3,60)=2.98$, $p=.04, \eta_{\mathrm{p}}^{2}=.13$.

Post-hoc tests based on the interaction between image type and retained range showed simple main effects of image type in the original, low-pass, middle-pass, and high-pass conditions, $F(1,20)>$ $56.58, p<.001, \eta_{\mathrm{p}}^{2}>.73$, for all conditions. The simple main effects of the retained range were significant in the trypophobic and control conditions, $F(3,60)>16.28, p<.001, \eta_{\mathrm{p}}{ }^{2}>.44$, for both conditions. Multiple comparisons with Shaffer's (1986) method demonstrated that in the trypophobic condition, the rating scores of the high-pass condition were significantly higher than those of the original, low-pass, and middle-pass conditions, $t(20)>6.28, p<.001$, Cohen's $d>1.87$, for all conditions. In the control condition, the rating scores of the original condition differed significantly from the low-pass, middle-pass, and high-pass conditions, $t(20)>4.46, p<.003$, Cohen's $d>0.93$, for all comparisons.

Moreover, post-hoc tests based on the interaction among the three factors revealed that in the trypophobic image, the simple main effects of the trypophobic-trait group were significant at the original, lowpass, and middle-pass conditions, $F(1,160)>6.92, p<.01, \eta_{\mathrm{p}}{ }^{2}>.25$, for all conditions, indicating that the rating scores were lower in the high TQ group than in the low TQ group. In the control condition, none of the simple main effects of the trypophobic-trait group were significant in all of the retained ranges, $F(1,80)<2.20, p>.14, \eta_{\mathrm{p}}{ }^{2}<.09$, for every retained range.

Furthermore, we conducted one-sample $t$ tests between 0 and the rating scores of the original, low-pass, middle-pass, and high-pass conditions on each of the image types. In the trypophobic condition, the rating scores of the original, low-pass, and middle-pass conditions differed significantly from $0, t(20)>8.03, p<.001$, Cohen's $d>2.37$, for all conditions, while the rating scores of the high-pass condition did not differ significantly from $0, t(20)=0.46, p=.64$, Cohen's $d=0.14$. In contrast, in the control condition, the rating scores of the original condition differed significantly from $0, t(20)=4.99, p<.001$, Cohen's $d$ $=1.49$, while those of the low-pass, middle-pass, and high-pass conditions did not differ significantly from $0, t(20)<1.02, p>.31$, Cohen's $d$ $<0.12$, for all conditions.

Experiment 3 successfully replicated the results of Experiment 2: The images in the original, low-pass, and middle-pass conditions caused comparable discomfort in the trypophobic condition. More importantly, the high TQ group experienced stronger discomfort from the original, low-pass, and middle-pass trypophobic images than the low TQ group, and there was no difference in discomfort from the high-pass trypophobic images between levels of the high and low TQ groups. One might claim that the differences in discomfort from the original, low-pass, and middle-pass trypophobic images between the high and low TQ groups were due to other factors apart from trypophobia that are coincidentally shared by the images. If this were plau- 
sible, we would have found a difference between the high and low TQ groups in the control condition, which was not the case. Thus, considering that people with a high level of the trypophobic trait tend to react to trypophobic images with stronger discomfort (Le et al., 2015), these results suggest that both the low- and mid-frequency components play key roles in trypophobic discomfort.

\section{GENERAL DISCUSSION}

The present study examined how the elimination of the spatial frequencies at each range influenced trypophobic discomfort. Experiment 1 showed that eliminating the mid-frequency components did not reduce trypophobic discomfort. Experiment 2 revealed that the images in the high-pass condition did not induce discomfort in the trypophobic condition, while the trypophobic images in the low-pass and middlepass conditions induced as much discomfort as the original condition did. In Experiment 3, participants with a high level of the trypophobic trait experienced stronger discomfort from the original, low-pass, and middle-pass trypophobic images than participants with a low level of the trypophobic trait. These findings suggest that trypophobic discomfort was based not only on mid-frequency but also on low-frequency components.

Cole and Wilkins (2013) conducted spectral analyses and revealed that mid-frequency components were involved in trypophobic discomfort. Experiment 2 showed that the images including only midfrequency components induced the same amount of discomfort in the trypophobic condition as the original images did. Additionally, trypophobic discomfort from the images including only mid-frequency components depended on the level of the trypophobic trait. These results were consistent with the findings of Cole and Wilkins. However, we showed that the images including only low-frequency components induced as much discomfort as the original images did, and the trypophobic trait modulated the discomfort from the images including only low-frequency components in the trypophobic condition. Like a previous study (Le et al., 2015), these results indicated that trypophobic discomfort was unlikely to be based solely on mid-frequency components and that low-frequency components also induced this discomfort.

How are the spatial frequencies of images related to perceived discomfort? We found that mid-frequency components induced trypophobic discomfort. A previous study has shown that the high energy at midrange spatial frequency is related to the visual discomfort induced by other types of images (Fernandez \& Wilkins, 2008). These findings suggest that mid-frequency components contribute to general visual discomfort. On the other hand, Cole and Wilkins (2013) have revealed that trypophobic images also have high-contrast energy at midrange spatial frequency. Additionally, Experiment 3 showed that discomfort from the middle-pass trypophobic image was dependent on the level of the trypophobic trait. Based on the previous and our findings, general visual discomfort might also be involved with trypophobic discomfort.

In addition to mid-frequency components, we have newly discovered that low-frequency components are important in trypophobic discomfort. This novel evidence possibly provides a further indication regarding the rapid detection of trypophobic objects. Based on recent neuroscientific evidence, it has been proposed that a colliculo-pulvinar pathway rapidly provides rough fear-related information to the amygdala (e.g., Vuilleumier, Armony, Driver, \& Dolan, 2003; Winston, Vuilleumier, \& Dolan, 2003). According to this proposal, when a fear-inducing stimulus is input, the fear-related cues of low spatial frequency are processed at the superior colliculus and pulvinar and this information is rapidly transmitted to the amygdala. This system results in instant and unconscious detection of fear-inducing threatening objects (e.g., Bar, Neta, \& Linz, 2006). The same might be true for trypophobic images because, as Experiments 2 and 3 revealed, lowfrequency components in the trypophobic images were able to evoke discomfort. Based on these ideas, we should detect trypophobic objects rapidly and unconsciously. However, the way in which low-frequency components in trypophobic images are involved in inducing discomfort remains unclear. Most trypophobic objects are nonpoisonous and harmless (such as lotus seedpods), and thus the ability to rapidly detect them seems unnecessary. Future studies should address these issues.

One might argue that the apparent frequency at some parts of the images meditated in the present results. In the present study, the image size was quite large $\left(20^{\circ} \times 20^{\circ}\right)$ and some parts of the image were inspected via peripheral vision. The receptive field of the periphery is larger than that of the fovea (e.g., Smith, Singh, Williams, \& Greenlee, 2001), and thus the apparent frequency in peripheral vision altered (e.g., Davis, Yager, \& Jones, 1987). Although we applied a Hanning Window to the stimuli, some peripheral information remained. Thus, in the present study, low-frequency components in the periphery might have been severed as mid-frequency components, thus possibly influencing the results of our experiments. It would be beneficial to examine this point in order to reveal which frequency, the apparent or physical, is important for inducing trypophobic discomfort.

The present study investigated the relationship between spatial frequency and trypophobic discomfort. It should be noted that cognitive processing might play an important role in trypophobic discomfort. Cole and Wilkins (2013) found that images of dangerous animals have the same visual properties as trypophobic images, which suggests that such images might induce some sort of aversion. That is, aversion might be the key cognitive factor of discomfort from clusters of holes. From the perspective of aversion, one cognitive mechanism underlying trypophobic discomfort is a defensive function. We benefit from mechanisms that help us to avoid potential threats, such as poisonous and harmful objects (Rozin \& Fallon, 1987). In this mechanism, visual cues are advantageous because we can avoid threats by staying away. Similar defensive functions have been discussed in pathogen or stranger avoidance. In the behavioral immune system (Park, Schaller, \& Crandall, 2007; Schaller, 2011; Schaller \& Park, 2011), perceptual cues of infectious pathogens elicit aversive emotions, and, in turn, these emotions facilitate behavioral avoidance. Likewise, the concept of stranger avoidance (Kawabe et al., 2017; Yamada et al., 2012, 2013, 2014) supposes that when objects are apparently uncertain and difficult to categorize into already acquired classes, we judge the objects as potentially harm- 
ful ones to be avoided. Consequently, negative reactions are elicited. As with pathogen or stranger avoidance, trypophobic images can be evaluated negatively and avoided because the appearance of clusters of holes can be associated with harmfulness, and we can immediately initiate avoidance behavior. Experimental investigations of these cognitive factors are essential for a comprehensive understanding of the mechanisms underlying trypophobic discomfort.

\section{FOOTNOTES}

${ }^{1}$ In the same way as the previous study (Cole \& Wilkins, 2013), we used a Bonferroni correction to adjust the significance level $(\alpha=$ $.004)$.

\section{AUTHOR NOTE}

Kyoshiro Sasaki and Yuki Yamada contributed equally to this work.

\section{ACKNOWLEDGMENTS}

The authors would like to thank Arnold Wilkins, Geoff Cole, and An Le for providing the images and the results of the spectral analyses. The authors would also like to express their appreciation to Kentaro Yamamoto, Ryota Akiho, and Fumiya Yonemitsu for collecting the data. The present study was supported by JSPS KAKENHI Grant Number JP14J06025 and 17J05236 given to K. S., JSPS KAKENHI Grant Numbers JP26540067 and JP15H05709 given to Y. Y., JSPS KAKENHI Grant Number JP16K04427 given to K. M., and Kyushu University Interdisciplinary Programs in Education and Projects in Research Development (\#27822) given to Y. Y.

\section{COMPETING FINANCIAL INTERESTS}

The authors declare no competing financial interests.

\section{REFERENCES}

Adobe Photoshop (Creative Suite 6) [Computer software]. San Jose, CA; Adobe Systems Incorporated.

Bar, M., Neta, M., \& Linz, H. (2006). Very first impressions. Emotion, 6, 269-278. doi: 10.1037/1528-3542.6.2.269 WWW

Brainard, D. H. (1997). The psychophysics toolbox. Spatial Vision, 10, 433-436. doi: 10.1163/156856897X00357 WWW

Caseras, X., Mataix-Cols, D., An, S. K., Lawrence, N. S., Speckens, A., Giampietro, V., . . Phillips, M. L. (2007). Sex differences in neural responses to disgusting visual stimuli: Implications for disgust-related psychiatric disorders. Biological Psychiatry, 62, 464-471. doi: 10.1016/j.biopsych.2006.10.030 WWW

Chaya, K., Xue, Y., Uto, Y., Yao, Q., \& Yamada, Y. (2016). Fear of eyes: Triadic relation among social anxiety, trypophobia, and discomfort for eye cluster. PeerJ, 4:e1942. doi: 10.7717/peerj.1942 WWW

Cole, G. G., \& Wilkins, A. J. (2013). Fear of holes. Psychological Science, 24, 1980-1985. doi: 10.1177/0956797613484937 WWW

Curtis, V., Aunger, R., \& Rabie, T. (2004). Evidence that disgust evolved to protect from risk of disease. Proceedings of the Royal
Society of London B: Biological Sciences, 271, S131-S133. doi: 10.1098/rsbl.2003.0144 wWw

Davis, E. T., Yager, D., \& Jones, B. J. (1987). Comparison of perceived spatial frequency between the fovea and the periphery. Journal of the Optical Society of America. A, 4, 1606-1611.doi:

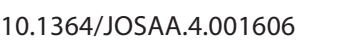

Fernandez, D., \& Wilkins, A. J. (2008). Uncomfortable images in art and nature. Perception, 37, 1098-1113. doi: 10.1068/p5814 WWW

Kawabe, T., Sasaki, K., Ihaya, K., \& Yamada, Y. (2017). When categorization-based stranger avoidance explains the uncanny valley: A comment on MacDorman \& Chattopadhyay (2016). |WWW Cognition, 161, 129-131. doi: 10.1016/j.cognition.2016.09.001

Le, A. T., Cole, G. G., \& Wilkins, A. J. (2015). Assessment of trypophobia and an analysis of its visual precipitation. Quarterly Journal of Experimental Psychology, 68, 2304-2322. doi: 10.1080/17470218.2015.1013970 |wWW

MATLAB [Computer software]. Natwick, MA: The MathWorks, Inc. O'Hare, L., \& Hibbard, P. B. (2011). Spatial frequency and visual discomfort. Vision Research, 51, 1767-1777. doi: 10.1016/j.

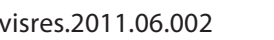

Park, J. H., Schaller, M., \& Crandall, C. S. (2007). Pathogenavoidance mechanisms and the stigmatization of obese people. Evolution and Human Behavior, 28, 410-414. doi: 10.1016/j. evolhumbehav.2007.05.008

Pelli, D. G. (1997). The Video Toolbox software for visual psychophysics: Transforming numbers into movies. Spatial Vision, 10, 437-442. doi: 10.1163/156856897X00366 (WWW

Rozin, P., \& Fallon, A. E. (1987). A perspective on disgust. Psychological Review, 94, 23-41. doi: 10.1037/0033-295X.94.1.23 WWW

Sawchuk, C. N., Lohr, J. M., Westendorf, D. H., Meunier, S. A., \& Tolin, D. F. (2002). Emotional responding to fearful and disgusting stimuli in specific phobics. Behaviour Research and Therapy, 40, 1031-1046. doi: 10.1016/S0005-7967(01)00093-6 WWW

Schaller, M. (2011). The behavioural immune system and the psychology of human sociality. Philosophical Transactions of the Royal Society of London B: Biological Sciences, 366, 3418-3426. doi: 10.1098/rstb.2011.0029 WWW

Schaller, M., \& Park, J. H. (2011). The behavioral immune system (and why it matters). Current Directions in Psychological Science, 20, 99-103. doi: 10.1177/0963721411402596

Shaffer, J. P. (1986). Modified sequentially rejective multiple test procedures. Journal of the American Statistical Association, 81, 826-831.

Smith, A. T., Singh, K. D., Williams, A. L., \& Greenlee, M. W. (2001). Estimating receptive field size from $\mathrm{fMRI}$ data in human striate and extrastriate visual cortex. Cerebral Cortex, 11, 1182-1190. doi: 10.1093/cercor/11.12.1182 WWW

Vuilleumier, P., Armony, J. L., Driver, J., \& Dolan, R. J. (2003). Distinct spatial frequency sensitivities for processing faces and emotional expressions. Nature Neuroscience, 6, 624-631. doi: 10.1038/nn1057 $\overline{\text { WWW }}$ 
Winston, J. S., Vuilleumier, P., \& Dolan, R. J. (2003). Effects of lowspatial frequency components of fearful faces on fusiform cortex activity. Current Biology, 13, 1824-1829. doi: 10.1016/j. cub.2003.09.038 WWW

Yamada, Y., Kawabe, T., \& Ihaya, K. (2012). Can you eat it? A link between categorization difficulty and food likability. Advances in Cognitive Psychology, 8, 248-254. doi: 10.2478/v10053-0080120-2 $\underline{W W W}$
Yamada, Y., Kawabe, T., \& Ihaya, K. (2013). Categorization difficulty is associated with negative evaluation in the "uncanny valley" phenomenon. Japanese Psychological Research, 55, 20-32. doi: 10.1111/j.1468-5884.2012.00538.x

Yamada, Y., Sasaki, K., Kunieda, S., \& Wada, Y. (2014). Scents boost preference for novel fruits. Appetite, 81, 102-107. doi: 10.1016/j. appet.2014.06.006 $\overline{\mathrm{WWW}}$

RECEIVED 19.10.2016| ACCEPTED 19.06.2017 